
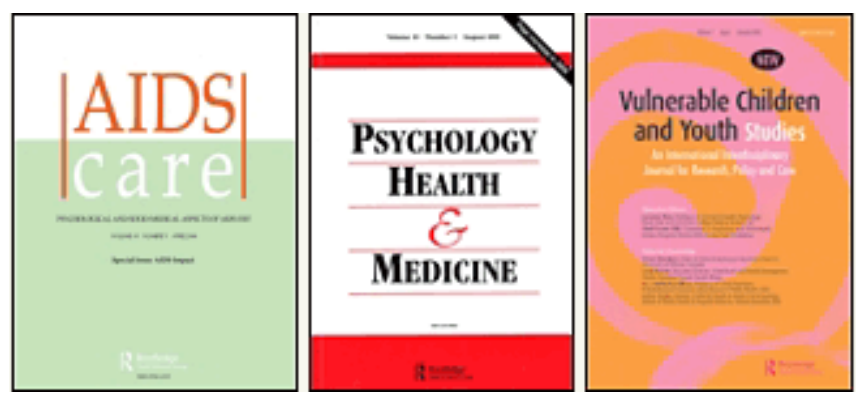

\title{
AIDSIMPACT SPECIAL ISSUE: WHEN GOOD NEWS IS BAD NEWS: PSYCHOLOGICAL IMPACT OF FALSE POSITVE DIAGNOSIS OF HIV
}

\begin{tabular}{|r|l|}
\hline Journal: & $\begin{array}{l}\text { AIDS Care - Psychology, Health \& Medicine - Vulnerable Children } \\
\text { and Youth Studies }\end{array}$ \\
\hline Manuscript ID: & AC-2007-08-0468.R1 \\
\hline Journal Selection: & AIDS Care \\
\hline Keywords: & HIV, false positive, Psychological impact \\
\hline \multicolumn{2}{|l}{} \\
\hline
\end{tabular}

\section{今 scholaroNE" \\ Manuscript Central}




\section{AIDSIMPACT SPECIAL ISSUE: WHEN GOOD NEWS IS BAD NEWS: PSYCHOLOGICAL IMPACT OF FALSE POSITVE DIAGNOSIS OF HIV}

\section{Abstract:}

HIV testing is known to be stressful, however impact of false positive HIV results on individuals is not well documented. This is a series of four case, who developed psychological difficulties and psychiatric morbidities, after being informed they had been misdiagnosed of HIV positive status. We look into documented cases of misdiagnosis and potential risks of misdiagnosis. The case series highlights the jmplications a false diagnosis HIV positive status can have, even when the diagnosis is rectified. Impact of misdiagnosis of HIV can lead to psychosocial difficulties and psychiatric morbidity, have public health and epidemiological implications and can lead to medico-legal conflict. This further reiterates the importance of HIV testing carried out ethically and sensitively, and in line with guidelines, respecting confidentiality and consent, and offering counselling pre-test and post-test, being mindful of the reality erroneous and false positive HIV test results. The implications of misdiagnosis are on the individual, their partners and social contacts, as well as for the community.

\section{Aim:}

To report the psychological impact of notification of the confirmed HIV negative status to people who had previously been diagnosed with HIV positive status. We Jooked into the potential sources of mistaken diagnosis with HIV testing and explored the impact of erroneous results from the psychosocial, epidemiological and medico-legal domains.

\section{Background:}

The UNAIDS/WHO 2006 AIDS Epidemic Update, with an estimated 39.5 million people are living with HIV [Global AIDS epidemic continues to grow]. There is jncreased initiative for HIV testing [e.g. WHO and UNAIDS issue new guidance on HIV testing and counselling in health facilities, 2007]. The United Nations and WHO published a policy statement in 2004, stating the importance of three- Cs in HIV testing-Confidentiality, Counselling, and Consent. In the United Kingdom both the General Medical Council ['Serious Communicable Diseases' 1997] and the Department of Health [Guidelines for pre-test discussion on HIV testing, 1996] had published documents with regards to HIV testing emphasizing both pre and post-test counselling.

HIV testing is taken seriously not only because of the epidemic nature and high mortality of the illness, but also the psychological impact of HIV testing itself. HIV-positive result js associated with psychological morbidity (Catalan 1995). This is acute after initial diagnosis (Leiberich 1997) with gradual adjustment over time (Pedersen and Elkit 1998). Diagnosis of HIV also affects relationships, occupation, social stigma and future planning. Suicidal ideation is associated with testing process of HIV. Though there is a reduction in suicidal ideation in testing negative, there was no increase in risk with testing positive. Suicidal ideation was

\begin{tabular}{|c|}
\hline Deleted: case \\
\hline Deleted: individuals \\
\hline $\begin{array}{l}\text { Deleted: in the context of current } \\
\text { methods of HIV testing }\end{array}$ \\
\hline Deleted: huge \\
\hline $\begin{array}{l}\text { Deleted: The implications of } \\
\text { misdiagnosis are on the individual, } \\
\text { their partners and social contacts, as } \\
\text { well as for the community. }\end{array}$ \\
\hline Deleted: also \\
\hline Deleted: such \\
\hline Deleted: . We \\
\hline Deleted: aspect \\
\hline Deleted: epidemiology \\
\hline $\begin{array}{l}\text { Deleted: HIV continues to be } \\
\text { identified as an epidemic by }\end{array}$ \\
\hline Deleted: $t$ \\
\hline $\begin{array}{l}\text { Deleted: WHO. According to the } \\
\text { latest figures published today in the }\end{array}$ \\
\hline Deleted: also rising concern and \\
\hline $\begin{array}{l}\text { Deleted: . The recent guidelines } \\
\text { published by Worth Health } \\
\text { Organization }\end{array}$ \\
\hline $\begin{array}{l}\text { Deleted: The document states that } \\
80 \% \text { of the people in 'low and } \\
\text { middle-income countries', who are } \\
\text { HIV positive, are unaware of their } \\
\text { serostatus. The document does not } \\
\text { recommend non-specific HIV } \\
\text { screening and does emphasize the } \\
\text { psychological impact of HIV testing. }\end{array}$ \\
\hline $\begin{array}{l}\text { Deleted: . It stresses the importance } \\
\text { of }\end{array}$ \\
\hline Deleted: accompanying \\
\hline Deleted: informed \\
\hline $\begin{array}{l}\text { Deleted: . Both these documents } \\
\text { speak of }\end{array}$ \\
\hline Deleted: The reason why \\
\hline $\begin{array}{l}\text { Deleted: and needs special } \\
\text { considerations is perhaps based }\end{array}$ \\
\hline Deleted: on \\
\hline $\begin{array}{l}\text { Deleted: from research, which } \\
\text { looked into }\end{array}$ \\
\hline Deleted: It has been noted \\
\hline Deleted: has been \\
\hline $\begin{array}{l}\text { Deleted: persistent psychiatric } \\
\text { difficulties }\end{array}$ \\
\hline Deleted: more \\
\hline $\begin{array}{l}\text { Deleted: and adaptation associated } \\
\text { with increased support }\end{array}$ \\
\hline $\begin{array}{l}\text { Deleted: has implications beyond the } \\
\text { medical domain and }\end{array}$ \\
\hline
\end{tabular}


found to persist with pre and post test counselling in over $15 \%$ of seropositive and seronegative individuals (Perry et al 1990).

We found a series of five cases_(Gill et al 1991) and few case reports of erronous HIV diagnosis (Vernon 1987, Zumwait et al 1987, Tyson 1987, Wu et al 1988). The risk of false positive results increases in low risk population screens (Meyer and Pauker 1987). $\underline{A}$ newspaper article referred to a man, found to HIV negative after fourteen months of being HIV positive (Gallagher, 2005). This is of relevance in screening blood donors (Altrah et al 1995). It is important that positive results are further investigated to establish a confirmed diagnosis (Mylonakis 2000).

There were no substantial data on the impact of notification of patients of false positive HIV positive status. One of the cases reported referred above (Vernon et al 1987) was noted to require psychotherapy for 'continued adjustment difficulties related to his misdiagnoses'.

A systemic review looking into long-term effect of false positive mammograms (Brewer et al 2007)found that false positive results impacted on health seeking behaviour of women There was one newspaper report of a man who committed suicide after being falsely diagnosed of carcinoma (Brockes, 2001). Another newspaper article, reported a man misdiagnosed with cancer, spent all his money (Tozer 2007). These reports reflect the complexity with which people can respond to a false diagnosis.

\section{Method:}

Information from four medico-legal reports of cases, with erroneous diagnosis of HIV positive status were summarized. We have kept personal information to the minimumaiming to respect confidentiality. We presented with psychological impact and psychiatric morbidity.pre and post-diagnosis ychiatric conditions, post-diagnosis and following revision of diagnosis to HIV negative status.

We carried out a literature search into the topic of psychological impact on people with misdiagnosis of $\mathrm{HIV}_{\mathbf{*}}$ We found minimal data in this regard. We found some data looking into impact of misdiagnosis in other conditions, We Jooked into psychological impact of HIV testing and potential sources of erroneous results. We carried out literature searches through the Ovid database using keywords of 'HIV testing', 'false positive', 'misdiagnosis' and 'psychological impact'. Relevant references from articles were searched. We also searched public domain data through generic Internet search engines.

\section{Case Reports:}

\section{Case-1:}


33-years-old Caucasian heterosexual woman was found to be HIV positive following investigations in 1991. She had past psychiatric contact with the adolescent services, and had suffered emotional difficulties in the context of relationship problems. She suffered from hyperthyroidism but did not have any ongoing psychiatric complains. She initially suffered from an adjustment disorder following the positive diagnosis. From 1993 to 1995 she suffered from depression with hypochondriacal reassurance seeking behaviour with fear of developing AIDS. She experienced rejection in her relationship and discrimination in her job. She then gradually came to terms with the condition by 1996 and positively adapted her social life leading to jmproved mental state.

In 1997 she was re-investigated and found to be HIV negative, at a time when she was attempting to return to paid employment. After an initial couple of weeks of elation following receipt of this information, she developed a depressive episode. This was associated with anger over her predicament and anxiety of losing her newfound social circle which was based around AIDS support groups.

\section{Case-2}

35-years-old Caucasian gay man was found to be HIV positive on a routine 6 monthly testing in 1996. He had past history of bulimia nervosa though his condition was on remission. His job involved direct health care delivery,

Following the diagnosis he suffered from depression with suicidal ideation. While struggling to cope, he engaged in risk taking behaviour (unprotected sex and harmful use of alcohol). He disclosed his HIV status and was moved to a non patient contact job, which he did not enjoy. In 1999 he was re-tested after no confirmatory test was found in his notes. He was found to be HIV negative. Initial elation and shock soon gave away to low mood needing treatment with anti-depressant medication. He experienced anger towards perceived wasted time in his life.

\section{Case-3}

25-years-old heterosexual Asian, who was a victim of torture seeking asylum was housed with a HIV positive person which made him anxious. Testing revealed positive HIV status in 1991.

Following the diagnosis he developed depression with obsessive symptoms. This gradually resolved but in 1993 he married under pressure from family. The marriage broke down within months due to his anxiety of transmitting HIV to his wife. He suffered severe adjustment disorder at this point. He suffered extreme shame and guilt at this ordeal. Thereafter he displayed resilience to reorganize his life and started working with victims of torture. In 1996 he developed a cold, which he believed was sign of imminent AIDS. On seeking medical advice discrepancies appeared between his history and medical records, which led to retesting. The results confirmed a HIV negative status. He experienced anger and shock at

\begin{tabular}{|l|}
\hline Deleted: child and \\
\hline Deleted: as a minor \\
\hline Deleted: seven years prior to \\
diagnosis \\
\hline $\begin{array}{l}\text { Deleted: and was on treatment } \\
\text { when diagnosed HIV positive }\end{array}$ \\
\hline Deleted: initial HIV \\
\hline Deleted: suffered \\
\hline Deleted: displayed courage and \\
motivation and \\
\hline Deleted: gradual improvement of \\
her
\end{tabular}

Deleted: suffered

Deleted: . This

Deleted: by then

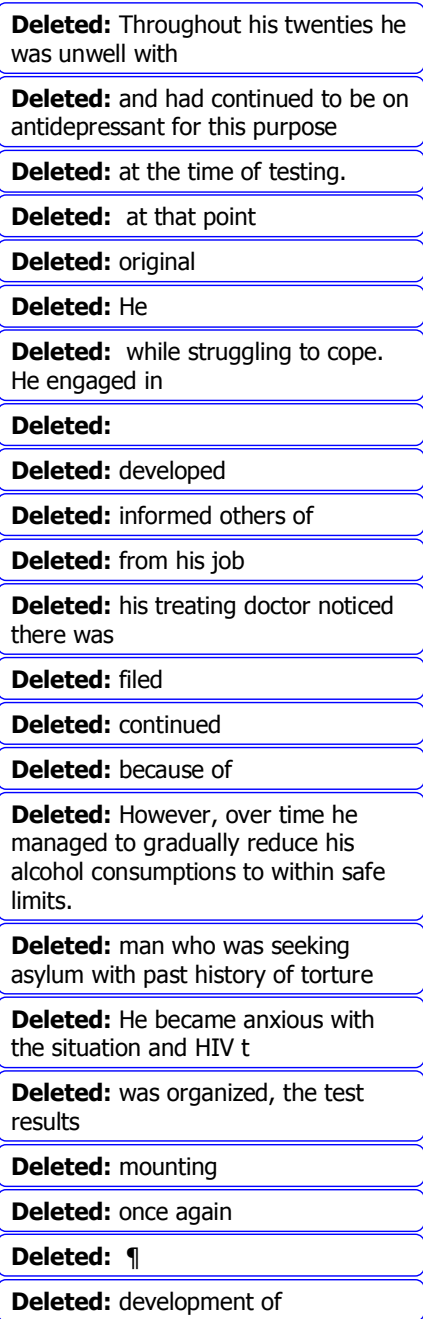


this development, and regretted loss of years of his life. He developed depression with labile and irritable affect with suicidal ideation and PTSD type 're-living' symptoms of past torture.

\section{Case-4}

19-years-old woman was diagnosed of HIV positive status after travelling abroad in 1989.

She had Anorexia Nervosa in her adolescence.

She developed acute stress reaction with hypochondriacal features, followed by mixed anxiety and depression She received psychotherapy and treatment with antidepressants. She modified her career choice presuming a short_ife expectancy. She remained in a stable relationship but experienced sexual dysfunction. She received private care between 1990 and 1999.

She was re-tested when she moved to NHS care in 1999 and was found to be HIV negative. She experienced anger and regret for major life decisions and developed Acute Stress Reaction and Panic Attacks. Her relationship also suffered with the change of roles and expectations.

\section{Comment:}

We observed a pattern of psychological impact following the disclosure of the previous erroneous result There was an initial period of shock and elation lasting couple of weeks followed by a more chronic response associated with anger and resentment over perceived wasted time and opportunities. There was stress, associated with readjusting and psychiatric morbidities such as depression, anxiety and panic attacks.

\section{Discussion:}

Table 1. Potential Causes of Erroneous Diagnosis of HIV positive status

\section{Serological Causes}

1. Interpreting Intermediate results

2. 'True' False Positive

3. Pregnancy

4. Neonates

5. Immunological conditions such as SLE, malignancy

6. Infections with immunological overlap such as HBV

7. Volunteers in vaccination trials

\section{Logistical Issues}

1. Human Error such as mislabelling samples, erroneous recording of results or misreporting to patient

2. Screening not followed by follow-up

3. Seeking [confidential] testing under false name

4. Factitious Disorders
Deleted: precious time

Deleted: . There was also

Deleted: emergence of

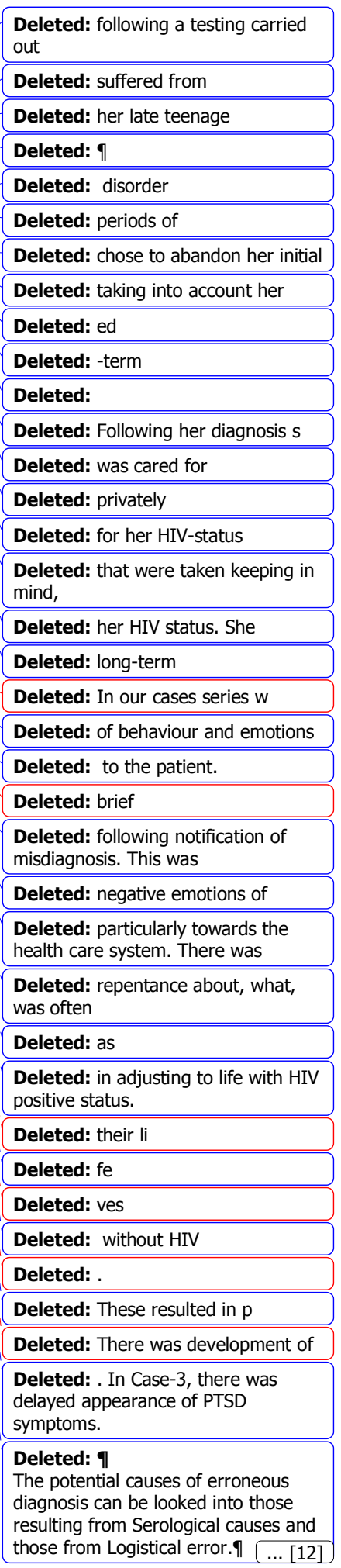


With screening ELISA test [Enzyme Immunoassay] there is a potential for misinterpretation jn determining the visual cut-off in a result (Kleinman 1998). With Western Blot test, often used as confirmatory test, there can be potential confusion in case of partial antigenic response, i.e. 'intermediate' results (Kleinman 1998). In such cases further testing in the form of Polymerase chain reaction (PCR) and or Nucleic Acid based tests (NAT) js recommended and tests repeated after three months. The 2006 UK National Guideline on document HIV testing claims: 'Observational data are limited, but in ten years of application of the 'three months rule' by all members of the UK HIV Laboratory Forum no reports of its failure have been received, and thus it is recommended that in general the three month rule continues to be applied'. It is worth noting in this context that all the cases in our study were diagnosed to be HIV positive before 1996.

Current diagnostic protocols in the western world offer extremely sensitive and specific diagnosis. False positive rates are believed to be between 0.0004 to $0.0007 \%$ while false negative rates of around $0.00 \underline{0} 3 \%$ (Kleinman 1998). However concern remains in cases where the entire diagnostic protocol is not followed through. It is known people with 'evasive style' of coping, are at increased risk of psychiatric morbidity associated with HIV testing (Leiberich 1997). It can be hypothesized these are the people who are at increased risk of not following through the entire diagnostic protocol. There are case reports of emotional trauma in patients who chose to avoid confirmatory testing after testing positive on screening [Correspondence JAMA 1993; AIDS letter 1999], and people who were not reassured even after repeated negative tests.

Physiological conditions such as pregnancy [Doran and Parra, 2000] and neonatal state [Owens et al 1996] sensitivity and specificity of tests are reduced. People suffering from Systemic Lupus Erythematosus (Sommer 2004) are at increased risk of testing false positive. People who had participated in HIV vaccine trials might present with a challenge in HIV testing (Weber 1997). The issue of serological false negativity is beyond the scope of this article.

The potential of false positive results magnify with less rigorous testing protocols. These would include testing in low and medium income countries where potentially due to resource constraints, more sophisticated tests may not routinely be available, to follow through, in all cases of ambiguous results.

The UNAIDS document on testing endorses voluntary testing, diagnostic testing in the symptomatic and testing in high-risk groups. It does not support but recognizes the presence of mandatory testing, such as testing associated with employment, or in certain countries joining armed forces or for immigration. These settings raise concerns surrounding the ethics
Deleted: Diagnosis of HIV is based on the $\mathrm{S}$

Deleted: . There is

Deleted: with ELISA testing

Deleted: For

Deleted: which is often

Deleted: to

Deleted: in case of

Deleted: needs to be carried out
Deleted: for whatever reason

Deleted: In cases where false positive or ambiguous results of initial tests do not get retested, followed through and confirmed.

Deleted: who

Deleted: after false positive tests

Deleted: Over the last ten years, in UK, positive tests are retested after 3 months.

Deleted: Certain conditions are known to increase the risk of false positivity. There are $p$

\section{Deleted: where}

Deleted: The 2006 UK National Guideline on document HIV testing claims: 'Observational data are

limited, but in ten years of application

of the 'three months rule' by all members of the UK HIV Laboratory

Forum no reports of its failure have

been received, and thus it is

recommended that in general the

three month rule continues to be

applied'. It is worth noting in this

context that all the cases in our study

were diagnosed to be HIV positive before 1996.9

Deleted: occurring under current protocols is rare. However in setting

of

Deleted: it can happen more frequently.

Deleted: perhaps

Deleted: test

Deleted: With increased impetus on mass testing this problem can magnify. $\emptyset$

Deleted: in certain countries

Deleted: to certain countries

Deleted: ethical

Deleted: 
of consent, confidentiality ${ }_{\perp}$ counselling and the level of sensitivity with which results are feedback, as well as the rigor of testing protocols in such situations.

The United States Food and Drug Administration first approved home kits in 1996. In 2006 the FDA clarified in a public statement that The Home Access Express HIV-1 is the only home-kit it approved Oraquick and Orasure are two other brands available in the UK. The approved home kits claim $100 \%$ sensitivity but we were not able to obtain information regarding risk of false positivity or false negativity. It is difficult to regulate the home kit market There are brands, which have been declared fraudulent (e.g. Globus Media of Canada, Lei-Home kit from Carolina, US), There are also ethical considerations surrounding effectiveness of counselling along with self-test kits, without face-to-face contact. In the UK the Terrance Higgins Trust supports public access to self-test kits, and the UK Government posted a technology note in this regard (Medical self test kits, 2003). In 1992 the government had banned HIV self test kits in UK.

There is increased emphasis on improving access to HIV testing, such as with public health and voluntary sectors (Dialogai in Geneva). There is an increasing risk of HIV testing being carried out without the rigor it deserves, and thereby increasing risk of error. Public health authorities are promoting testing and initiating policies based on sero-status (e.g. serosorting). These can have immense public health implications in all countries.

\section{Conclusion:}

With recent increased emphasis on HIV testing as evident from the WHO document, there is a need for increased awareness both among public health authorities as well as in individuals of the reality of false positive test results and potential for error in diagnosis. We need to be mindful of the ethics behind HIV testing and ensure application of the rigorous testing protocols. Diagnosing someone as HIV positive has huge implications in medical, psychological and social domains for the individual. It has implications in terms of epidemiology As obvious from the very basis of our study ${ }_{\perp}$ there are medico-legal implications for the health care provider testing for HIV. There are also medico-legal implications for the individual in terms of insurance and disclosure.

\section{References:}

Altrah, H.I., et al (1995) Management of Blood Donors whose donations are repeatedly falsely positive by HIV antibody screening, Journal of Clinical Pathology, 48, 865-867

Brewer, N.T., et al (2007) Systemic review: The Long term Effects of False Positive Mammograms, Annals of Internal Medicine. 146(7), 502-510

Brockes, E., (13.01.2001) Death after false cancer diagnosis, The Guardian, 10

Catalan, J., (1995) Psychological Interventions in Infection with the Human Immunodeficiency Virus. The British Journal of Psychiatry, Volume 167(1), pp 104-111
Deleted: and

Deleted: . There are also issues surrounding rigorousness

Deleted: these settings, e.g. if

screening tests are not followed

through with confirmatory tests

Deleted: There are also issues surrounding the level of sensitivity with which results are feedback.

Deleted: There is increasing demand and availability of home-kits for HIV testing.

Deleted: This has led to the United States Food and Drug Administration (

Deleted: ) releasing a

Deleted: in this regard (FDA 2006). The FDA first approved home kits in 1996.

Deleted: $a$

Deleted: by FDA

Deleted: However i

Deleted: and $t$

Deleted: which have been

fraudulent

Deleted: though

Deleted: in 1992

Deleted: Certain conditions are known to increase the risk of false positivity. There are physiological conditions such as pregnancy [Doran and Parra, 2000] and neonates and infants [meta-analysis by Owens et al 1996] where sensitivity and specificity of tests are reduced. People suffering from Systemic Lupus Erythematosus (Sommer 2004) are at increased risk of testing false positive. There are people who have participated in HIV vaccine trials who might present with a challenge in HIV testing (Weber

1997). The issue of serological false negativity is beyond the scope of this study. $\emptyset$

Deleted: There needs to be increased rigor at being

Deleted: about the three components

Deleted: HIV testing, i.e. consent confidentiality and pre and post test counselling

Deleted: also

Deleted: both in the context of false positivity as well as sero-sorting techniques being promoted among HIV positive population in certain countries 
Doran, T. I., Parra, E., (2000) False-Positive and Indeterminate Human Immunodeficiency Virus Test Results in Pregnant Women, Archives of Family Medicine ;9:924-929

Gallagher, I., (13 ${ }^{\text {th }}$ November 2005) First Man in World, The Mail on Sunday, 6

Gill, J.M., et al (1991) Five cases of erroneously diagnosed HIV infection, Canadian Medical Association Journal, 1593-1595

Global AIDS epidemic continues to grow, (2006), World Health Organization, http://www.who.int/hiv/mediacentre/news62/en/index.html

Guidelines for pre-test discussion on HIV testing, (1996), Department of health, United Kingdom,

http://www.dh.gov.uk/en/Publicationsandstatistics/Lettersandcirculars/Professionalletters/Chi efofficerletters/DH 4004038

James, T., ( $7^{\text {th }}$ May 2007) The Good news: Doctor got it wrong and I am not dying The Bad news: I spent all my money on a farewell spree, Daily Mail, 21

Kleinman, S., et al (1998) False Positive HIV-1 test results in a low risk setting of voluntary blood donation. Retrovirus Epidemiology Donor Study, Journal of the American Medical Association 280(12) 1080-1085

Leiberich, P., (1997) Longitudinal Development of Distress, Coping and Quality of Life in HIVpositive persons, Psychother Psychosom, 66(5) 237-247

Marzuk, P.,(1991) Suicidal behaviour and HIV illnesses. International Review of Psychiatry, 3, 365-371

Mayer, K.B., and Pauker, S.G., (1987) Screening for HIV; Can we Afford false positive rate, The New England Journal of Medicine, 238-240

Medical self test kits (2003) Parliamentary Office of Science and Technology, http://www.parliament.uk/post/pn194.pdf

Myolanakis, E., et al (2000) Report of False Positive HIV test Result and the Potential use of Additional tests in Establishing HIV serostatus, Archive of Internal Medicine, 160, 2386-2388 Owens, D. K., (1996), A meta-analytic evaluation of the polymerase chain reaction for the diagnosis of HIV infection in infants, JAMA, 275(17), 1342-1348

Pedersen, S.S., Elkit, A., (1998) Traumatisation, Psychological defence style, coping, symptomatology in HIV -positive: A pilot study, Scandinavian Journal of Psychology, 39(2): $55-60$

Perry, S., Jacobsberg, L., Fishman, B., et al (1990) Psychiatric diagnosis before serological testing for HIV. American Journal of Psychiatry, 147, 89-93.

Perry, S., Jacobsberg, L., \& Fishman, B., (1990) Suicidal ideation and HIV testing. Journal of the American Medical Association, 263, 679-682.

Rogstad, K. E., et al, (2006) United Kingdom National Guidelines on HIV Testing 2006, Clinical Effectiveness Group: British Association of Sexual health and HIV, www.bashh.org/guidelines/2006/hiv testing june06.pdf

Serious Communicable Diseases ,(1997), General Medical Council, http://www.gmcuk.org/guidance/current/library/serious_communicable diseases.asp

| Sommer. S., (2004), Systemic lupus erythematosus or infection with HIV, or both? Clinical and Experimental Dermatology, 29 ( 4 ) 393-395 
Testing Yourself for HIV-1, the Virus that Causes AIDS (April 2006) U.S. Food and Drug Administration, http://www.fda.gov/cber/infosheets/hiv-home2.htm

Tyson, E., et al, (1987) Fraudulent AIDS A Variant of Munchausen's Syndrome, 258, 20632064

UNAIDS/WHO Policy Statement on HIV testing, June 2004, UNAIDS and WHO http://www.who.int/rpc/research_ethics/hivtestingpolicy_en_pdf.pdf

Weber, J., (1997) Distinguishing between Response to HIV Vaccine and Response to HIV, Lancet, 350 (9073), 230-231

WHO and UNAIDS issue new guidance on HIV testing and counselling in health facilities, 30 MAY 2007, World Health Organization, http://www.who.int/mediacentre/news/releases/2007/pr24/en/index.html

Wu, A., et al (1988) Factitious False positive for HIV, Journal of American Medical Association, 259(11), 1647

Zumwait, R.E., (1987) Fraudulent AIDS, Journal of American Medical Association, 257, 3231 
Page 2: [1] Deleted rahul bhattacharya

11/6/2007 11:18:00 AM

There have been case reports and case series on false positive results for HIV.

Page 2: [1] Deleted

There is a published rahul bhattacharya 11/6/2007 11:16:00 AM

\section{Page 2: [1] Deleted} case

rahul bhattacharya

11/6/2007 11:17:00 AM

Page 2: [1] Deleted rahul bhattacharya

11/6/2007 11:18:00 AM of erroneous diagnosis of HIV in 1991

\section{Page 2: [1] Deleted} rahul bhattacharya

11/6/2007 11:18:00 AM

Page 2: [1] Deleted rahul bhattacharya

11/6/2007 11:18:00 AM There are

Page 2: [1] Deleted other false

rahul bhattacharya

11/6/2007 11:19:00 AM

Page 2: [1] Deleted

rahul bhattacharya

11/6/2007 9:34:00 AM

Difficulty in diagnosing HIV status confidently in certain populations has been identified.

\section{Page 2: [1] Deleted} blood

$$
\text { rahul bhattacharya }
$$

11/6/2007 11:20:00 AM

Page 2: [1] Deleted rahul bhattacharya

11/6/2007 11:20:00 AM in low prevalence areas

\section{Page 2: [2] Deleted reports}

rahul bhattacharya

11/6/2007 9:35:00 AM

Page 2: [2] Deleted rahul bhattacharya

11/6/2007 9:35:00 AM of false positive result

\section{Page 2: [2] Deleted} rahul bhattacharya

11/6/2007 11:22:00 AM There was a newspaper article referring to a man, found to HIV negative after fourteen months of being HIV positive (Gallagher, 2005). This can be an unconfirmed case of false positive status or misdiagnosis and at the time of the report he was in legal battle over the issue of diagnosis.

Page 2: [3] Deleted user 11/14/2007 3:36:00 PM Looking into the impact of misdiagnosis of a chronic life threatening conditions we came across a

Page 2: [3] Deleted user 11/14/2007 3:36:00 PM . The study 
We also came across a

Page 2: [4] Deleted rahul bhattacharya

$11 / 6 / 2007$ 11:27:00 AM which looked into the

Page 2: [4] Deleted rahul bhattacharya $11 / 6 / 2007$ 11:27:00 AM negative social and financial impact in

\begin{tabular}{lcc}
$\begin{array}{l}\text { Page 2: [4] Deleted } \\
\text { person }\end{array}$ & rahul bhattacharya & $\mathbf{1 1 / 6 / 2 0 0 7}$ 11:26:00 AM \\
\hline $\begin{array}{l}\text { Page 2: [4] Deleted } \\
\text { Who then }\end{array}$ & rahul bhattacharya & $\mathbf{1 1 / 6 / 2 0 0 7}$ 11:27:00 AM \\
\hline $\begin{array}{l}\text { Page 2: [4] Deleted } \\
\text { as a consequence }\end{array}$ & rahul bhattacharya \\
\hline $\begin{array}{l}\text { Page 2: [4] Deleted } \\
\text { notification and highlights instances where this can have potential grave impact of such an event. }\end{array}$
\end{tabular}

Page 2: [5] Deleted
Described below is a case series of four cases known from

Page 2: [5] Deleted user 11/14/2007 3:42:00 PM contact

Page 2: [5] Deleted user 11/14/2007 3:43:00 PM associated

Page 2: [6] Deleted user 11/14/2007 3:43:00 PM

. Information on the cases was obtained from their medico-legal report.

Page 2: [7] Deleted rahul bhattacharya

11/6/2007 11:30:00 AM prepared by one of the authors [Cases 2, 3 and 4] and by another psychiatrist [Case -1 ].

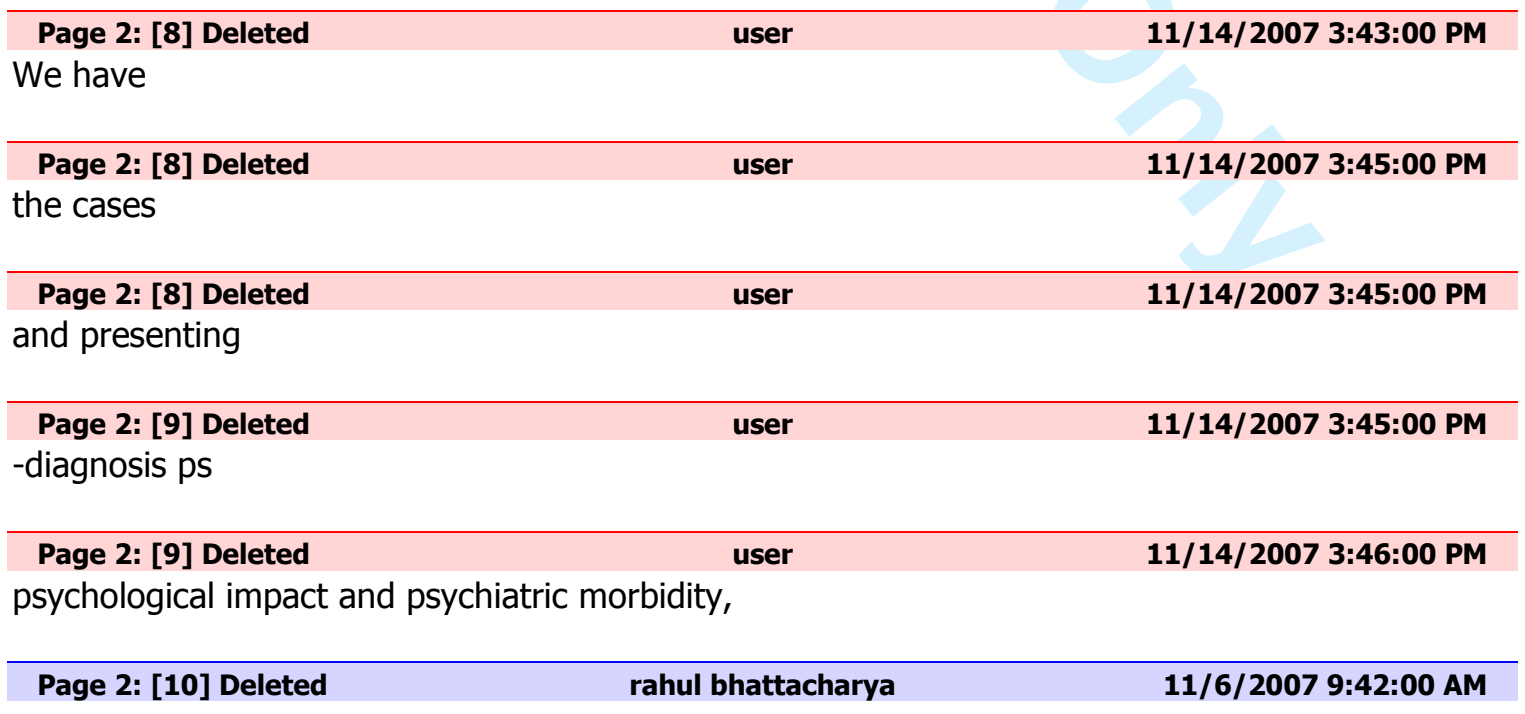


then

Page 2: [10] Deleted of informing

Page 2: [10] Deleted patient of

\section{Page 2: [10] Deleted} positive status.

Page 2: [11] Deleted and diagnosis

Page 2: [11] Deleted under current diagn

Page 2: [11] Deleted for

Page 4: [12] Deleted rahul bhattacharya

rahul bhattacharya

rahul bhattacharya

rahul bhattacharya

rahul bhattacharya
$11 / 6 / 2007$ 11:32:00 AM

11/6/2007 9:43:00 AM

$11 / 6 / 2007$ 11:32:00 AM

$11 / 6 / 2007$ 11:31:00 AM

11/6/2007 11:32:00 AM

$11 / 6 / 2007$ 11:33:00 AM
$11 / 6 / 200711: 33: 00$ AM

11/6/2007 10:10:00 AM

The potential causes of erroneous diagnosis can be looked into those resulting from Serological causes and those from Logistical error. 